\title{
Non-contact voltage relay for switching windings of a boost transformer
}

\author{
E.G. Usmanov ${ }^{1}$, A.N. Rasulov ${ }^{1}, M . K$. Bobojanov ${ }^{1}$, R.Ch. Karimov ${ }^{1 *}$ \\ ${ }^{1}$ Tashkent State Technical University named after Islam Karimov, 100095, Uzbekistan, Tashkent, University St. 2A
}

\begin{abstract}
In power supply systems the change in the magnitude of the voltage degrades the operation of electrical appliances right up to their failure. The proposed scheme of a contactless voltage relay based on a contactless voltage relay with switching the windings of a booster step-up transformer with a time delay allows switching the windings of a magnetizing booster transformer with a voltage change at the consumer terminals. Analytical studies of a non-autonomous non-linear dynamic circuit, which is the basis of the proposed device based on the classical method of solving a differential equation, which determines the nature of the voltage change for controlling thruster switches. The change of energy parameters of the proposed stabilizer depending on the voltage is given. The article considers the principle of operation of the proposed scheme of a contactless switching device based on a contactless voltage relay that switches the windings of a booster step-up transformer. An analytical study of a non-autonomous nonlinear dynamic circuit, which is the basis of the proposed device and the characteristics $u_{c}=f t$ required for switching the thruster is presented.
\end{abstract}

One of the important indicators of the quality of electricity is the stability of the current voltage value. Using special technical means of regulation namely transformers with voltage regulation under load or stepup transformers it is possible to improve the quality of the voltage. In this case, it is necessary to change the number of turns the primary windings of the transformer or switch the magnetizing windings of the amplifier amplifier transformers. For this purpose we propose to use contactless voltage relays based on resistive nonlinear circuits [1-3].

Consider the circuit (Fig.1) of the proposed contactless switching device based on an optoelectronic voltage relay. The device consists of two proximity relays. The first (I) relay is used to turn on the voltage boost transformer (BT) winding. The second (II) relay serves to disconnect the winding of the boost transformer.

The proposed circuit works as follows: when the input voltage is reduced the first relay is activated and a control signal is applied to open the power thruster, which turns on the winding of the boost transformer in the network. With a further increase in the input voltage, the second voltage relay is triggered by shunting the optocoupler diode circuit of the first relay with its thruster optocoupler circuit terminating the access of the control signal to the power thruster; such tripping of the power thruster is still achieved as soon as the load current passes through zero, thereby discon-necting the boost coil transformer [4-5].

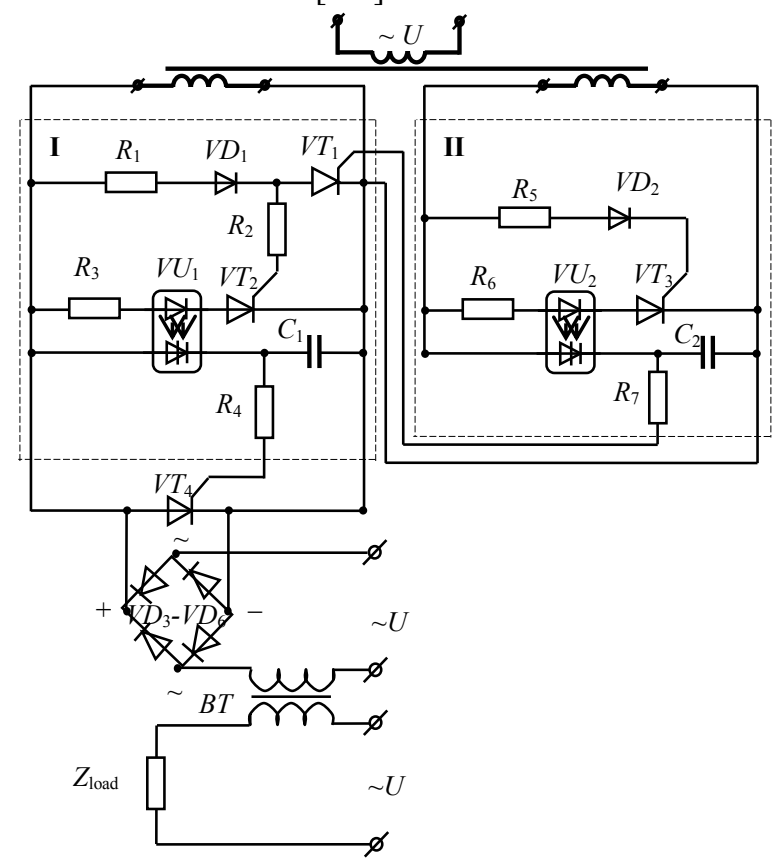

Fig.1. Diagram of a non-contact optoelectronic voltage relay for switching windings of a boost transformer

When creating non-contact voltage relays with a sinusoidal form of voltage at the load a non-autonomous non-linear dynamic circuit consisting of a diode $V D_{1}$,

* Corresponding author: raxmatillo82@mail.ru 
active resistance $R_{1}$, capacitor $C$ and resistance $R_{2}$ is used. Also on the output side there are optocouplers $V U$, diode bridge $V D_{2}-V D_{5}$ and, load $Z_{\text {load }}$ (Fig.2) [6-7, 9].

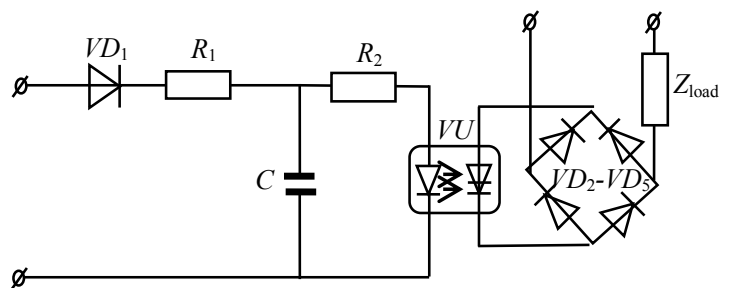

Fig.2. Non-autonomous non-linear dynamic circuit

Currently, various methods of analysis of such chains are widely used. For a theoretical analysis the equation of state of the circuit shown in Fig.2, it is proposed to use the numerical Euler method. In this case it is necessary to determine an approximate solution of the equation on a certain interval $[6,12]$.

Denote by:

$$
\frac{d y}{d t}=f(t, y)
$$

$$
t_{1}-t_{0}=t_{2}-t_{1}=\cdots=t_{n}-t_{n-1}=\Delta t=h
$$

From expression (2), each point $t_{0} t_{1} t_{2} \ldots t_{n}$ is replaced by the differences in the relations of the derivative, then:

$$
\Delta y=f(t, y) \Delta t
$$

In the first section, $y_{1}-y_{0}=\Delta y$ and $\Delta t=h$, hence

$$
y_{1}-y_{0}=f t_{0}, y_{0} h
$$

From (4) it is known $y_{0}, t_{0} h$, then:

$$
y_{1}=y_{0}+f t_{0}, y_{0} h
$$

At $t=t$, from equation (3) we get:

$$
\Delta y=f t, y h
$$

From here,

$$
y_{2}-y_{1}=f t_{0}, y_{0} h
$$

Then,

$$
y_{2}=y_{1}+f t_{1}, y_{1} h
$$

For the remaining sections of the equation is written similarly,

$$
\begin{gathered}
y_{3}=y_{2}+f t_{2}, y_{2} h \\
y_{n}=y_{n-1}+f t_{n-1}, y_{n-1} h
\end{gathered}
$$

Thus, we determine the approximate values of the points $t_{0}, t_{1}, t_{2}, \ldots t_{n}$ of the function " $\mathrm{y}$ " connecting these points, we obtain linear Euler curves.

We analyze this chain using the classical method [1, 12]. We take the characteristic of the diode as ideal and assume that $u=U_{m} \sin \omega t$. Then from the moment $t_{0}$ to $t_{1}$ the diode is open and the circuit equation has the following form:

$U_{m} \sin \omega t=R_{1} i+U_{c}$,

Given that $i_{c}=C \frac{d u_{c}}{d c}$ we have:

$$
U_{m} \sin \omega t=R_{1} \quad c \frac{d u_{c}}{d t}+\frac{U_{c}}{R_{2}}+U_{c}
$$

From here,

$$
\frac{d u_{c}}{d t}=\frac{1}{R_{1} C} \quad U_{m} \sin t-U_{c}\left(1+\frac{R_{1}}{R_{7}}\right.
$$

Equation (11) is solved by the numerical Euler method

From here,

$$
U_{c(k+1)}=U_{c k}+f U_{c k} * t_{k} * h
$$

$$
\mathrm{f}\left(U_{c k}, t_{k}\right)=\frac{1}{R_{1} C} U_{m} \sin \omega t-U_{c} \quad 1+\frac{R_{1}}{R_{2}}
$$

Here $k=0,1,2, \ldots ; h$ - is the integration step.

Until $t=t_{1}$, the voltage across the capacitance is determined by (12), taking into account the initial conditions. From the moment $t=t_{1}$ the diode opens and until the moment $t_{2}$ the voltage on the capacitor remains at the voltage level for the moment $t_{2}$, the voltage on the capacitor remains at the voltage level for the moment $t_{1}$, from the moment $t_{2}$ the diode opens again and the voltage on the capacitance is again described by dependences (12) with other initial condition.

Figure 3-5 shows the curves of the voltage across the capacitance for various values of the circuit parameters at $U_{m}=$ Const and the integration step $h=0,001$ [11-12].

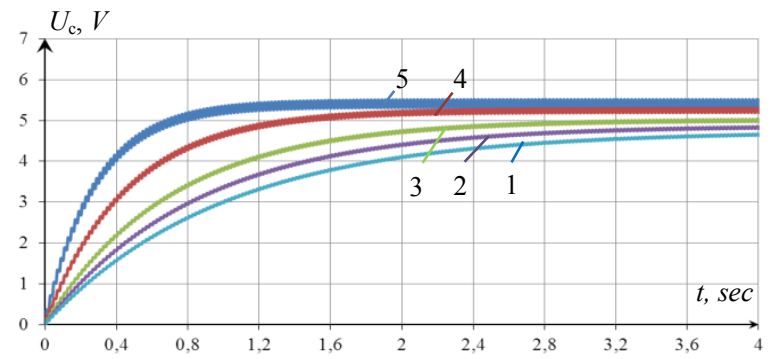

Fig.3. A family of curves of variation of voltage across a capacitance as a function of time at $C=1 \mu \mathrm{F} ; R_{2}=3,0$ $\mathrm{kOhm}$, for resistance values $R_{1}: 1$ - 0,3 kOhm; 2 - 0,5 kOhm; 3 - 0,8 kOhm; 4 - 1,0 kOhm; 5 - 1,2 kOhm

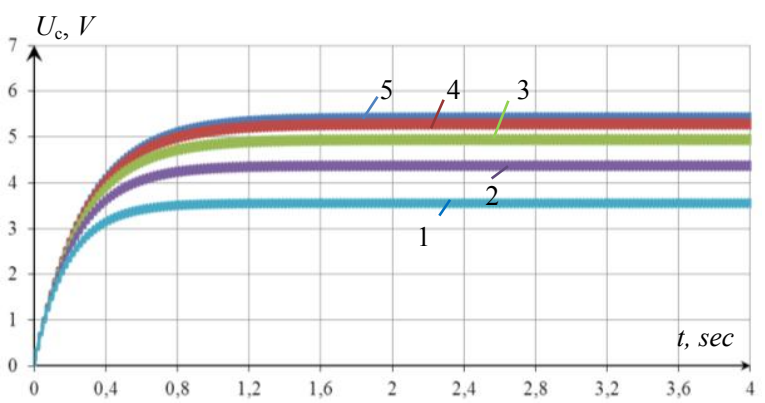

Fig.4. A family of curves of variation of voltage across a capacitance as a function of time at $C=1 \mu \mathrm{F} ; R_{1}=3,0$ $\mathrm{kOhm}$ for resistance values $R_{2}$ : 1 - 3,0 kOhm; 2 - 2,0 kOhm; 3 - 1,0 kOhm; 4 - 0,5 kOhm; 5 - 0,25 kOhm

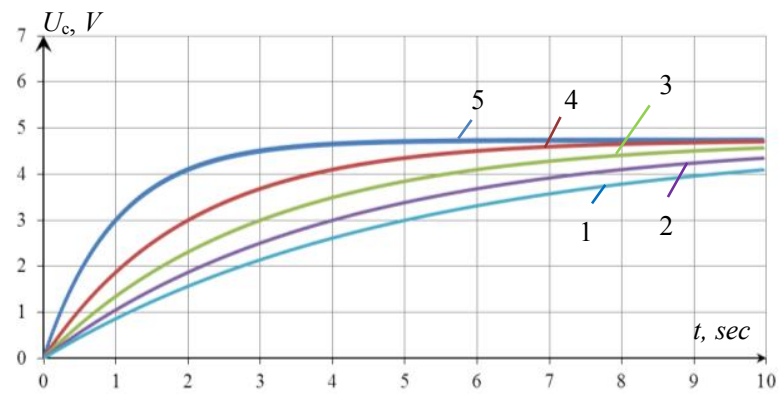

Fig.5. A family of voltage variation curves for capacitance as a function of time at $R_{1}=2,0 \mathrm{kOhm}$; $R_{2}=3,0 \mathrm{kOhm}$, for capacitance C: 1 - 1,0 $\mu \mathrm{F} ; 2$ - 2,0 $\mu \mathrm{F} ; 3-3,0 \mu \mathrm{F} ; 4-4,0 \mu \mathrm{F} ; 5-5,0 \mu \mathrm{F}$

It can be seen from the curves that by changing the parameters $R_{1}$ and $C$ of the circuit in question, it is possible to adjust within relatively large limits, the time the capacitor is fully charged, which is necessary for the LEDs of the optocoupler shown in Fig.2, to reduce the false response of the relay during switching changes in the supply voltage. 
Figure 6 shows the voltage change across the capacitance of a dynamic circuit during simulation in the Matlab Simulink package.

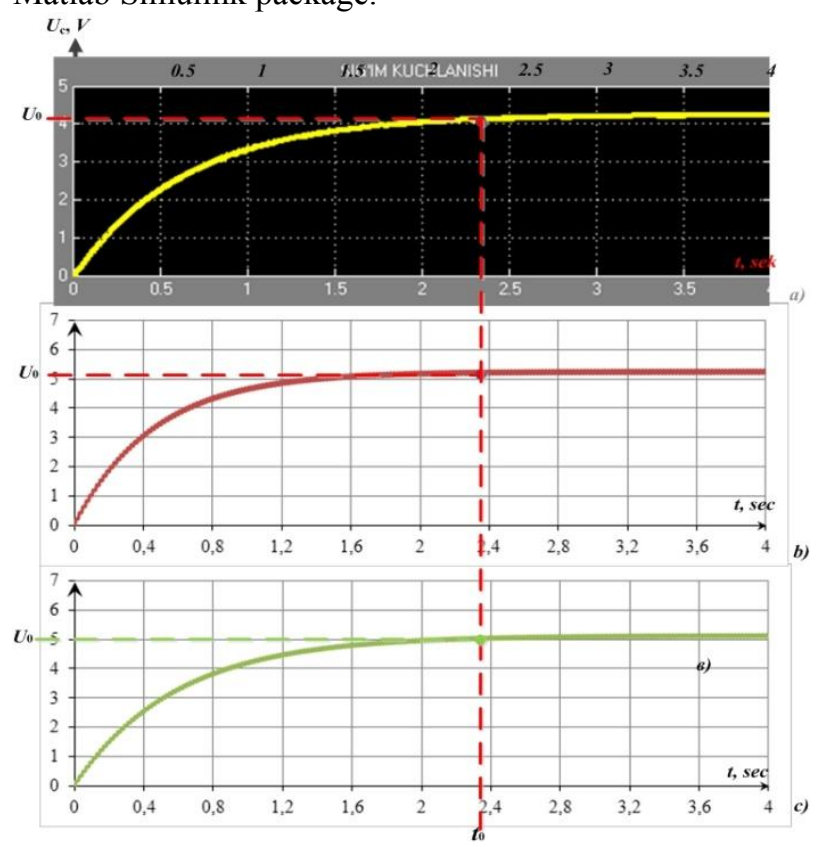

Fig.6. Comparative analysis of a nonlinear electric circuit based on modeling: $a$ - in the Matlab Simulink package; $b$ - Electronics Workbench; $c$ - a curve built on the basis of theoretical research

A comparative analysis of the dependences $U_{c}=$ $f t$ (Fig.6) shows that the difference in characteristics is $1.5-2.0 \%$. This indicates that the used method of theoretical research is acceptable for this chain [7-8, 10].

Fig.7 shows the waveforms of the voltage inputoutput voltage stabilizer. With $U_{\text {input }}=175 \mathrm{~V}$ (voltage deviation from the nominal $-20,4 \%), U_{\text {output }}=214 \mathrm{~V}$ ($2,72 \%)$. With $U_{\text {input }}=241 \mathrm{~V}(+8,71 \%), U_{\text {output }}=224 \mathrm{~V}$ $(+1,78 \%)$. Thus, when the input voltage varies from $20,4 \%$ to $+8,71 \%$ of the nominal value using the proposed device, we obtain the output voltage in the range from $-2,7 \%$ to $+1,7 \%$ of the nominal voltage, that meets the required standards.

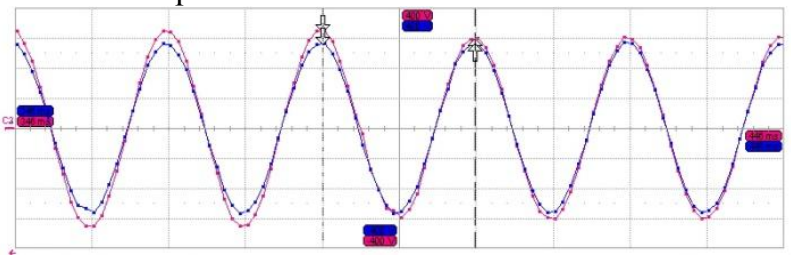

a) $U_{\text {input }}=175 \mathrm{~V} ; U_{\text {output }}=214 \mathrm{~V}$

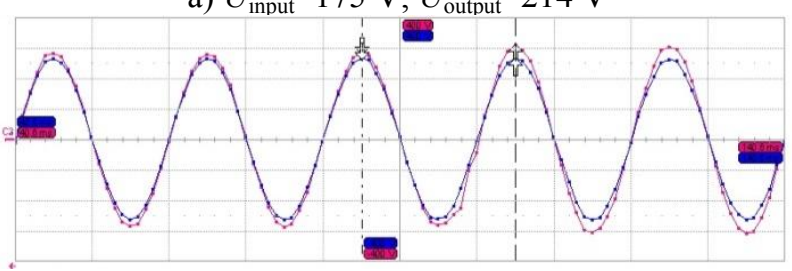

b) $U_{\text {input }}=241 \mathrm{~V} ; U_{\text {output }}=224 \mathrm{~V}$

Fig.7. Waveform of voltage input-output voltage stabilizer

Figure 8 shows the main energy indicators of the boost booster transformer. These characteristics show that when the load changes over a wide range the value of energy indicators changes within $\cos \varphi=0,96$; $\eta=0,8 \div 0,95$.

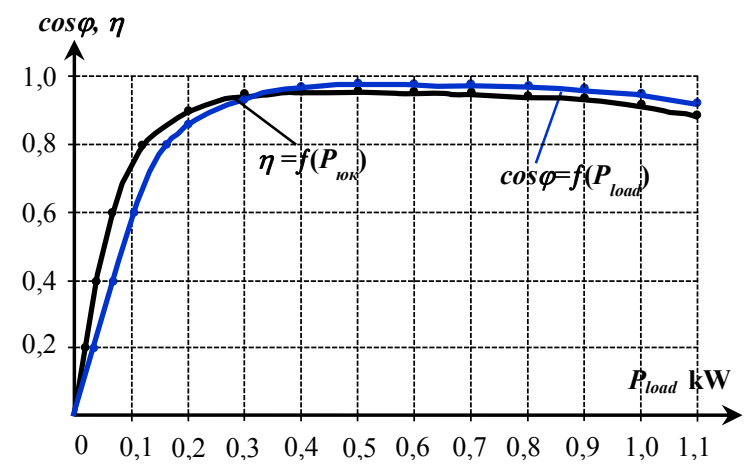

Fig.8. Characteristics of the dependence of $\cos \varphi$ and the coefficient of performance of the boost voltage transformer

\section{Conclusion}

The investigated voltage stabilizer circuit provides voltage stability of consumers within acceptable limits of $\pm 5 \%$ of the nominal value with a change in the input voltage within $175 \div 241 \mathrm{~V}$.

The control of the windings of the boost booster transformer using non-contact switching show high energy characteristics.

The proposed voltage relay circuit provides noncontact switching of the boost transformer windings when the input voltage changes over a wide range the output voltage has a sinusoidal shape and varies within \pm $2 \%$ of the rated voltage.

\section{References}

1. EG Usmanov, EH Abduraimov, RCh Karimov, Bulletin of TSTU, 3-4, 48-51 (2012).

2. RCh Karimov, Collection of materials of the IV international scientific conference "Modern Trends in Technical Sciences", 30-33 (2015).

3. EG Usmanov, EH Abduraimov, RCh Karimov, Problems of computer science and energy, 2-3, 37-41 (2006).

4. MK Samoxvalov, Textbook, UISTU,126 (2003).

5. MK Bobojanov, EG Usmanov, EH Abduraimov, RCh Karimov, JESR, 1-2, 210-212 (2018).

6. RCh Karimov, ICh Karimov, JYS USA, 5, 106-109 (2016).

7. UD Karix, 107 (2002). http://www/fineprint/com/

8. RCh Karimov, JESR, 9-10, 144-146 (2015).

9. RCh Karimov, Collection of materials of the IV international scientific conference "Modern Trends in Technical Sciences", 33-35 (2015).

10. AN Rasulov, RCh Karimov, JEE, 4, 179-183 (2015). 11. MQ Bobojanov, RCh Karimov, Bulletin of TSTU, 4(101), 53-57 (2017).

12. RCh Karimov, EG Usmonov, JA Xolbekov, Bulletin of TSTU, 2(107), 75-82 (2019). 